TSUKUBA J. MATH.

Vol. 10 No. 1 (1986). 63-72

\title{
ON THE REPRESENTATION TYPE OF LOCALLY BOUNDED CATEGORIES
}

\author{
By \\ Piotr Dowbor and Andrzej SkowroŃSKi
}

\section{Introduction}

In $[5,6,7]$ Drozd showed that the class of finite dimensional algebras over an arbitrary algebraically closed field splits into two disjoint subclasses: representation-tame algebras whose indecomposable modules of arbitrary fixed finite dimension may be parametrized by several discrete and several continuous parameters and representation-wild algebras for which the classification of indecomposable finite dimensional modules includes the classical unsolved problem on the canonical form of pairs of matrices with respect to the simultaneous conjugacy. From this point of view it would be interesting to have methods determining whether a given algebra is representation-tame. In many cases the covering techniques, introduced by Bongartz-Gabriel [3], Gabriel [8], Green [10] and Riedtmann [14] for the research of representation-finite algebras and developed recently by the authors [4] for the investigation of arbitrary algebras, reduce the classification problem of indecomposables to the corresponding problem for locally bounded categories, being infinite dimensional algebras without unity. Since the supports of finite dimensional representations of locally bounded categories form their finite subcategories it is natural to ask about a connection between the representation types of these categories.

In this paper we show that a locally bounded category is representation-tame if and only if any its finite full subcategory is representation-tame. In particular any locally bounded category is either representation-tame or representation-wild. Moreover different equivalent definitions of representation-tamness are presented here. These results have found some applications in $[4,15]$.

\section{The representation type of locally bounded categories.}

Throughout the paper $K$ will denote a fixed algebraically closed field. We recall some basic definitions of [3]: A $K$-category $R$ is a category whose morphism sets $R(x, y)$ are endowed with $K$-vector space structures such that the composition

Received September 17, 1984. Received July 9, 1985. 
of maps is $K$-bilinear. A locally bounded category is a $K$-category $R$ satisfying the following conditions:

(a) For each $x \in R$, the endomorphism algebra $R(x, x)$ is local.

(b) Distinct objects of $R$ are not isomorphic.

(c) For each object $x$ of $R, \sum_{y \in R}[R(x, y): K]<\infty$ and $\sum_{y \in R}[R(y, x): K]<\infty$. In the sequel $R$ denotes a fixed connected locally bounded category over $K$. An $R$ module is a $K$-linear contravariant functor from $R$ to $\operatorname{Mod} K$, where $\operatorname{Mod} K$ is the category of $K$-vector spaces. For any $R$-module $M$ we shall denote by supp $M$ the support of $M$, that is, the full subcategory of $R$ formed by all objects $x$ such that $M(x) \neq 0$. The dimension-vector of an $R$-module $M$ is the family $\operatorname{dim} M$ $=(M(x): K)_{x \in R}$, and $\operatorname{dim} M$ is finite if the dimension $\operatorname{dim} M=\sum_{x \in R}[M(x): K]$ is finite. We denote by $\operatorname{Mod} R$ the category of all $R$-modules and by $\bmod R$ (resp. ind $R$ ) the full subcategory of Mod $R$ formed by all finite dimensional $R$-modules (resp. finite dimensional indecomposable $R$-modules). For a finite dimension-vector $d$ in $R$ we will denote by ind $R$ the full subcategory of ind $R$ formed by all modules $M$ with $\operatorname{dim} M=d$. In the paper we will use the analogous notation $\operatorname{Mod} A, \bmod$ $A$, ind $A \operatorname{ind}_{n} A$ for a finitely generated $K$-algebra $A$ and $n \in N=\{1,2,3, \cdots\}$.

A parametrizing triple $(A, B, Q)$ over $R$ consists of a finitely generated $K$ algebra $A$, a full subcategory $B$ of $\bmod A$ and an $A$-R-bimodule $Q$. The restriction $-\left.\underset{A}{\otimes} Q\right|_{B}: B \longrightarrow \operatorname{Mod} R$ of the functor $-\underset{A}{\otimes} Q: \bmod A \longrightarrow \operatorname{Mod} R$ to $B$ is called the parametrizing functor associated with $(A, B, Q)$. Moreover, a full subcategory $D$ of mod $R$ is parametrized by a family of parametrizing triples $\left(A_{i}, B_{i}, Q_{i}\right), i \in I$, provided every module $M$ from $D$ is of the form $M=X_{i} \otimes Q_{i}$ for some $i \in I$ and $X_{i}$ from $B_{i}$. A functor $F: C \longrightarrow D$ is called strict (see [7, p. 244]) if $F(X) \cong F(Y)$ implies $X \cong Y$ for any objects $X$ and $Y$ from $C$. Finally, for a full subcategory $C$ of $R$, we will denote by $\hat{C}$ the full subcategory of $R$ formed by all objects $x$ such that $R(x, y) \neq 0$ or $R(y, x) \neq 0$ for some $y$ from $C$ (see [4]). Observe that if $C$ is finite then $\hat{C}$ is so.

Following Drozd $[5,6,7]$ a locally bounded category $R$ is called representationwild if there exists a strict functor $F: \bmod K\langle x, y\rangle \longrightarrow \bmod R$, where $K\langle x, y\rangle$ is the free associative $K$-algebra in two non-commuting variables, satisfying the following condition:

(1) $F=-\underset{K\langle x, y\rangle}{\otimes} Q$, where $Q$ is a $K\langle x, y\rangle-R$-bimodule being a finitely generated free left $K\langle x, y\rangle$-module. From the Drozd's proof of [6, Theorem 1] it follows that a finite locally bounded $K$-category $R$ is representation-wild if and only if there exists a strict functor $F: \bmod K\langle x, y\rangle \longrightarrow \bmod R$ satisfying (1) and the following stronger condition

(2) $F$ preserves indecomposables. 
Further, $R$ is called representation-tame if for any finite dimension-vector $d$ in $R$ the category $\operatorname{ind}_{d} R$ is parametrized by a finite family of parametrizing triples $\left(A_{i}, B_{i}, Q_{i}\right), i=1, \cdots, n_{d}$, such that $A_{i}$ are polynomial algebras $K[T]$ in one variable, $Q_{i}$ are finitely generated free left $A_{i}$-modules and $B_{i}=\operatorname{ind}_{1} K[T]$ are the categories of all simple right $K[T]$-modules. By a rational algebra we mean the localization $K[T]_{h}$ of $K[T]$ with respect to powers of a polynomial $h$ in $K[T]$.

The following proposition gives equivalent conditions for $R$ to be representation-tame.

Proposition 1. Let $R$ be a locally bounded $K$-category and let $d$ be a finite dimension-vector in $R$. The following equivalences hold:

(i ) $\operatorname{ind}_{d} R$ is parametrized by a finite family of parametrizing triples $\left(A_{i}, B_{i}, Q_{i}\right)$, $i=1, \cdots, n_{d}$, where all $A_{i}$ are polynomial algebras $K[T]$ in one variable, $Q_{i}$ are finitely generated free left $A_{i}$-modules and $B_{i}=\operatorname{ind}_{1} A_{i}$.

(ii) $\operatorname{ind}_{d} R$ is parametrized by a finite family of parametrizing triples $\left(A_{i}, B_{i}, Q_{i}\right)$, $i=1, \cdots, u_{d}$, such that $A_{i}$ are rational algebras $K[T]_{h_{i}}, Q_{i}$ are finitely generated left $A_{i}$-modules and $B_{i}=$ ind $A_{i}$.

(iii) ind $_{d} R$ is parametrized by a finite family of parametrizing triples $\left(A_{i}, B_{i}, Q_{i}\right)$, $i=1, \cdots, t_{d}$, such that $A_{i}=K[T]_{h_{i}}, Q_{i}$ are finitely generated free left $A_{i}$-modules and $B_{i}=$ ind $A_{i}$.

(iv) $\operatorname{ind}_{d} R$ is parametrized by a finite family of parametrizing triples $\left(A_{i}, B_{i}, Q_{i}\right)$, $i=1, \cdots, r_{d}$, such that $A_{i}=K[T]_{h_{i}}, Q_{i}$ are finitely generated free left $A_{i}$-modules and $B_{i}=\operatorname{ind}_{1} A_{i}$.

(v) $\operatorname{ind}_{d} R$ is parametrized by a finite family of parametrizing triples $\left(A_{i}, B_{i}, Q_{i}\right)$, $i=1, \cdots, s_{d}$, such that $A_{i}=K$ or $A_{i}=K[T]_{h_{i}}, Q_{i}$ are finitely generated free left $A_{i^{-}}$ modules, $B_{i}=\operatorname{ind}_{1} A_{i}$, and the parametrizing functors $F_{i}: B_{i} \longrightarrow \bmod R$ associated to $\left(A_{i}, B_{i}, Q_{i}\right)$ preserve indecomposables.

For the proof of this proposition we need some lemmas.

Lemma 1. Let $A$ be a rational algebra $K[T]_{h}$ and let $m \in N$. Then there exists an $A$-A-bimodule $P^{m}$ being a free left $A$-module of rank $m$ such that the restriction of $-\underset{A}{\otimes} P^{m}: \bmod A \longrightarrow \bmod A$ to $\operatorname{ind}_{1} A$ is a strict dense functor $\operatorname{ind}_{1} A \longrightarrow \operatorname{ind}_{m} A$.

Proof. Let $P^{m}$ be the free left $A$-module $\left({ }_{A} A\right)^{m}$ endowed with the right $A$ module structure such that the multiplication by $T$ is given by the right multiplication by the following $m x m$-matrix 


$$
\left(\begin{array}{llllll}
T & & & 0 & \\
1 & T & & & \\
& 1 & \cdot & & & \\
& 0 & \cdot & \cdot & \cdot & \\
& 0 & & 1 & T
\end{array}\right)
$$

It is not hard to see that $A /(p) \underset{A}{\otimes} P^{m} \cong A /\left(p^{m}\right)$ for any prime element $p$ in $A$ and we are done.

Lemma 2. Let $A$ be a rational algebra $K[T]_{h}$ and let $Q$ be an $A-R$-bimodule being a finitely generated left A-module. Then there exists an $A$-R-bimodule $Q^{\prime}$ which is a finitely generated free left A-module and the following condition holds: for each finite dimension-vector $d$ in $R$ there exists only finitely many nonisomorphic indecomposable $R$-modules of the form $X \underset{A}{\otimes} Q, X \in$ ind $A$, which are not isomorphic to a module $Y \underset{A}{\otimes} Q^{\prime}$ for some $Y \in$ ind $A$.

Proof. First observe that the $A$-submodule $t(Q)$ of ${ }_{A} Q$ consisting of all $A$ torsion elements of ${ }_{A} Q$ is an $R$-submodule of $Q_{R}$, and consequently is an $A-R$ subbimodule of $Q$. Indeed, $t(Q)=\oplus t(Q(x))$ and for any morphism $\mathcal{L}: x \longrightarrow y$ in $R, Q(\mathcal{L}): Q(y) \longrightarrow Q(x)$ is a homomorphism of left $A$-modules and then $Q(\mathcal{L})(t(Q(y)))$ is contained in $t(Q(x))$. Put $Q^{\prime}=Q / t(Q)$. Then ${ }_{A} Q^{\prime}$ is a finitely generated torsionfree left $K[T]_{h}$-module, so it is a finitely generated free left $K[T]_{h}$-module and ${ }_{A} Q={ }_{A} t(Q) \oplus{ }_{A} Q^{\prime}$. Hence for any indecomposable finite dimensional right $A$-module $Y$ we have the following dimension formula $\underline{\operatorname{dim}} Y \otimes_{A} Q=\underline{\operatorname{dim}} Y \bigotimes_{A} t(Q)+m\left(\boldsymbol{r}_{x}\right)_{x \in R}$, where $Y=A /\left(p^{m}\right)$ for some prime element $p \in A, m \in N$, and $r_{x}$ is the rank of the left free $A$-module $Q^{\prime}(x), x \in R$. Let $p_{1}, \cdots, p_{s}$ be all prime elements of $A$ such that $A /\left(p_{i}^{m_{i}}\right)$ is a direct summand of ${ }_{A} t(Q)$ for some $m_{i} \in N$. Then for any $Y=A /\left(p^{m}\right), p \neq p_{1}, \cdots$, $p_{s}, m \in N$, holds $Y \otimes t(Q)=0$ and we have an isomorphism $Y \otimes Q \cong Y \otimes Q^{\prime}$. Now let $d$ be a finite dimension-vector in $R$. We claim that there ${ }^{A}$ is only finitely many right $A$-modules of the form $X=A /\left(p_{i}^{m}\right), i=1, \cdots, s, m \in N$, such that $\operatorname{dim} X \otimes Q=d$. Indeed, as a consequence of the above dimension formula, for any $x \in \operatorname{supp} \widehat{Q}_{R}^{\prime}$, we get the inequality $d_{x}=\operatorname{dim} X \underset{\boldsymbol{A}}{\otimes} Q(x) \geqslant m r_{x}$ which gives a bound for $m$. The lemma is proved.

Lemma 3. Let $A$ be a rational algebra $K[T]_{h}$ and let $Q$ be an $A$-R-bimodule being a finitely generated free left $A$-module. Then there exists a $K[T]$-R-bimodule $Q^{\prime}$ being a finitely generated free left $K[T]$-module such that for any left $A$-module $Y$ the $R$-modules $Y \underset{A}{\otimes} Q$ and $Y \otimes_{K[T]} Q^{\prime}$ are isomorphic.

Proof. We will use arguments due to Drozd [5, Remark 2, p.109]. Let $Q^{\prime \prime}$ 
be a $K[T]$-submodule of the torsion-free left $K[T]$-module ${ }_{A} Q$ generated over $K[T]$ by some choosen $A$-basis of ${ }_{A} Q$. Denote by $Q^{\prime}$ the $R$-submodule of $Q_{R}$ generated by $Q^{\prime \prime}$, that is, the $R$-module given by $Q^{\prime}(x)=\sum_{y \in R} \sum_{\mathcal{L} \in R(x, y)} Q(\mathcal{L})\left(Q\left(1_{y}\right) Q^{\prime \prime}(y)\right), \quad x \in R$. Then $Q^{\prime}$ is again a finitely generated left $K[T]$-module. Indeed, $Q$ is a finitely generated free left $A$-module, so supp $Q$ is a finite locally bounded category and ${ }_{K[T]} Q^{\prime}$ can be presented as a sum of $m$ finitely generated left $K[T]$-modules where $m=\sum_{x, y \in \operatorname{supp} Q}[R(x, y): K]$. On the other hand, ${ }_{K[T]} Q^{\prime}$ is free as a submodule of the torsion-free $K[T]$-module ${ }_{A} Q$. Consequently $Q^{\prime}$ is an $K[T]$-R-subbimodule of ${ }_{K[T]} Q_{R}$ being a finitely generated free left $K[T]$-module. Finally the required isomorphisms $Y \underset{A}{\otimes} Q \cong Y \underset{K[T]}{\otimes} Q^{\prime}$ are a consequence of the $A$-R-bimodule isomorphism $Q \cong A \underset{K[T]}{\otimes} Q^{\prime}$.

Let $\Gamma=\left(\Gamma_{0}, \Gamma_{1}\right)$ be a locally finite quiver, I an twosided ideal in the quiver category $K \Gamma$ [9] and $C=K \Gamma / I$ the corresponding bounded quiver category. For any finite dimension-vector $d=\left(d_{x}\right)_{x \in \Gamma_{0}}$ in $C$ we denote by $D_{C}(d)$ the closed subset of the affine space $\sum_{(x \stackrel{\mathcal{L}}{\rightarrow} y) \in \Gamma_{1}} M_{d_{y} x d_{x}}(K)$, where $M_{d_{y} x d_{x}}(K)$ is the set of all $d_{y} x d_{x}$ matrices over $K$, formed by all tuples $\left(\lambda_{\mathcal{L}}\right)_{\mathcal{L} \in \Gamma_{1}}$ such that for any element $\sum_{r=1}^{n} a_{r} \mathcal{L}_{j r}^{(r)}$ $\cdots \mathcal{L}_{j_{1}}^{(r)} \in I(x, y)$, the $d_{y} x d_{x}$-matrix $\sum_{r=1}^{n} a_{r} \lambda_{j_{r}}^{(r)} \cdots \lambda_{j_{1}}^{(r)}$ is zero (see $[2,12]$ ). In this case $D_{C}(d)$ is called the variety of $C$-modules of dimension-vector $d$.

Let $A=K\left\{T_{1}, \cdots, T_{n}\right\}$ be a finitely generated $K$-algebra, considered as the corresponding bounded quiver algebra [9] of the quiver having one point and $n$ loops. Let $Q$ be an $A$ - $R$-bimodule being a finitely generated free left $A$-module and let $r=\left(r_{x}\right)_{x \in R}$ be the finite dimension-vector in $R$ such that $r_{x}$ is the rank of the free left $A$-module $Q(x)$. Then the functor $-\underset{A}{\otimes} Q: \bmod A \longrightarrow \bmod R$ induces a family of regular maps $q^{n}: D_{A}(n) \longrightarrow D_{R}(n r)$. Indeed, fix for any $x \in R$ an $A$-basis of the free left $A$-module $Q(x)$. Then for any arrow $\mathcal{L}: y \longrightarrow x$ in the ordinary quiver $Q_{R}^{*}$ of $R$ (see [9]), $Q(\mathcal{L}): Q(x) \longrightarrow Q(y)$ is the right multiplication by the matrix $\left(f_{i j}^{\mathcal{L}}\right) \in M_{r_{x} x r_{y}}(A)$, where $M_{r_{x} x r_{y}}(A)$ denotes the set of all $r_{x} x r_{y}$-matrices with coefficients in $A$. Now if $\lambda=\left(\lambda_{1}, \cdots, \lambda_{n}\right)$ is an element of $D_{A}(n)$ defining an $A$-module $Y=\left(K^{n}, \lambda\right)$, then for any arrow $\mathcal{L}: y \longrightarrow x$ in $Q_{R}^{*},(Y \otimes Q)(\mathcal{L}):\left(K^{n}\right)^{r} x \longrightarrow\left(K^{n}\right)^{r} y$ is the right multiplication by the matrix $q_{\mathcal{L}}^{n}(\lambda)=\left(f_{i j}^{\mathcal{L}}\left(\lambda_{1}, \cdots, \lambda_{n}\right)\right)$. Thus the map $q^{n}$ : $D_{A}(n) \longrightarrow D_{R}(n r)$ given by the formula $q^{n}(\lambda)=\left(q_{\mathcal{L}}^{n}(\lambda)\right)_{\mathcal{L} \in\left(Q_{R}^{*}\right)_{1}}$, where $\left(Q_{R}^{*}\right)_{1}$ the set of arrows of $Q_{R}^{*}$, is a regular map. For $n=1$ we will write $q$ instead of $q^{1}$.

LEMMA 4. Let $A$ be a finitely generated commutative $K$-algebra without nilpotents and let $d=\left(d_{x}\right)_{x \in R}$ be a finite dimension-vector in $R$. Then for any regular map $g: D_{A}(1) \longrightarrow D_{R}(d)$ there exists an $A$-R-bimodule $Q$ being a finitely generated free left $A$-module with $d_{x}=\operatorname{rank} Q(x), x \in R$, such that $g$ is the map $q$ induced by $Q$. 
Proof. From Hilbert's theorem [11, Corollary 1.4] $A$ is isomorphic to the algebra $K\left[D_{A}(1)\right]$ of regular functions on $D_{A}(1)$. We define the required $A-R$ bimodule as follows: for any $x \in R$ we set $Q(x)=\left({ }_{A} A\right)^{d} x$ and, for any arrow $\mathcal{L}: y$ $\longrightarrow x$ in $Q_{R}^{*}$ (the ordinary quiver of $R$ ), $Q(\mathcal{L}): Q(x) \longrightarrow Q(y)$ is the right multiplication by the matrix $\left(g_{i j}^{\mathcal{f}}\right) M_{d_{x} x d_{y}}\left(K\left[D_{A}(1)\right]\right)$, where $g_{i j}^{f}$ are given by $g$. Observe that defined above representations of the quiver $Q_{R}^{*}$ are annihilated by the ideal defining $R$ and hence $Q$ is an $A-R$-bimodule. The fact that $g$ is equal to the map $q$ induced by $Q$ is an immediate consequence of the definitions of $Q$ and $q$.

We have the following consequence of the above lemma.

CoROllaRy 1. Let $V$ be an affine variety, $d$ a finite dimension-vector in $R$ and $g: V \longrightarrow D_{R}(d)$ a regular map. Then there exists an $A$-R-bimodule $Q$ being a finitely generated free left A-module with rank $Q(x)=d_{x}$ such that $g$ is the map $q$ : $D_{K[V]}(1) \longrightarrow D_{R}(d)$ induced by $Q$.

Lemma 5. Let $A$ be a rational algebra $K[T]_{h}$ and let $Q$ be an A-R-bimodule being a finitely generated free left A-module. Assume also that the set $U$ of isoclasses of all indecomposable $R$-modules of the form $S \otimes Q$, for $S \in \operatorname{ind}_{1} A$, is infinite. Then there exists an element $h^{\prime}$ in $A$ such that the set of all isoclasses of $R$-modules of the form $S^{\prime} \underset{A^{\prime}}{\otimes} Q^{\prime}$ for some $S^{\prime} \in \operatorname{ind}_{1} A^{\prime}$, where $A^{\prime}=A_{h^{\prime}}$ and $Q^{\prime}=A^{\prime}{\underset{A}{Q} Q}_{Q}$, coincides with $U$.

Proof. Denote by $d_{x}$ the rank of the free left $A$-module $Q(x)$ and by $M_{d_{x}}$ the variety of all $d_{x} \times d_{x}$-matrices with coefficients in $K$. Then $d=\left(d_{x}\right)_{x \in R}$ is a finite dimension-vector in $R$ and let $Z$ be the subset of $D_{R}(d) \times \prod_{x \in R} M_{d_{x}}$ consisting of all pairs $(M, V)$ such that $V$ is an endomorphism of the $R$-module $M$ with properties $V^{2}=V$ and $V \neq 1_{M}, 0_{M}$. Let $p: D_{R}(d) \times \prod_{x \in R} M_{d_{x}} \longrightarrow D_{R}(d)$ be the canonical projection. Since $Z$ is locally closed in Zariski topology, by Chevalley theorem [11, Chap. II, p. $94,3.18,3.19]$ the subset $p(Z)$ of $D_{R}(d)$, consisting of all decomposable $R$-modules with dimension-vector $d$, is constructible. Let $V(h)$ be the set of zeros of $h,\left(K^{1}\right)_{h}$ $=K^{1} \backslash V(h)$, and $f:\left(K^{1}\right)_{h} \longrightarrow D_{R}(d)$ the regular map induced by $Q$, where we identify $D_{A}(1)$ with $\left(K^{1}\right)_{h}$. Since $f^{-1}(p(Z))$ is constructible and by our assumption $\left(K^{1}\right)_{h} \backslash f^{-1}(p(Z))$ is infinite, the set $f^{-1}(p(Z))$ is finite. Hence the polynomial $h^{\prime}=$ $\prod_{t \in p-1(p(Z))}(T-t)$ satisfies the required property.

REMARK 1. Let $M$ be a finite dimensional $R$-module and let $A$ be a finitely generated $K$-algebra. Then the $A$-R-bimodule $Q=A \underset{K}{\underset{K}{ } M}$ is a finitely generated free left $A$-module and $M=S \underset{A}{Q} Q$ for any $S \in \operatorname{ind}_{1} A$. 
Proof of Proposition 1. The implication (i) $\longrightarrow$ (ii) is obvious. (ii) $\longrightarrow$ (iii). Assume that $\operatorname{ind}_{d} R$ is parametrized by a finite family of parametrizing triples $\left(A_{i}, B_{i}, Q_{i}\right), 1 \leqslant i \leqslant n_{d}$, such that $A_{i}$ are rational algebras $K[T]_{h_{i}}, Q_{i}$ are finitely generated as left $A_{i}$-modules and $B_{i}=$ ind $A_{i}$. By Lemma 2 there exists a finite family of $K[T]$ - $R$-bimodules $Q_{i}^{\prime}, 1 \leqslant i \leqslant r_{d}, r_{d} \leqslant n_{d}$, such that all but a finite number of nonisomorphic modules of $\operatorname{ind}_{d} R$ are in the image of one of the parametrizing functors $F_{i}:$ ind $K[T] \longrightarrow \bmod R$ associated to the parametrizing triples $(K[T]$, ind $\left.K[T], Q_{i}^{\prime}\right), i=1, \cdots, r_{d}$. But from Remark 1 we can complete this set of triples to a finite family of parametrizing triples satisfying (iii).

(iii) $\longrightarrow$ (iv). Let $\left(A_{i}, B_{i}, Q_{i}\right), i=1, \cdots, t_{d}$, be a finite family of parametrizing triples satisfying (iii). For any $1 \leqslant i \leqslant t_{d}, x \in R$, denote by $r_{x}^{i}$ the rank of the left free $A_{i}$ module $Q_{i}(x)$ and put $r^{i}=\left(r_{x}^{i}\right)_{x \in R}$. Observe that if $Y_{i} \in \operatorname{ind}_{n_{i}} A_{i}$, then $\operatorname{dim} Y_{i} \otimes Q_{i}=$ $n_{i} r^{i}=\left(n_{i} r_{x}^{i}\right)_{x \in R}$. For any $i$, let $m_{i}$ be the natural number satisfying the equality $m_{i} r^{i}=d$ if such a number exists or $m_{i}=1$ in the opposite case. Then by Lemma 1 , $\operatorname{ind}_{d} R$ is parametrized by the family of triples $\left(A_{i}, \operatorname{ind}_{1} A_{i}, P^{m_{i}} \underset{A_{i}}{\otimes} Q_{i}\right), i=1, \cdots, t_{d}$, satisfying (iv).

(iv) $\longrightarrow(\mathrm{v})$. Assume that $\left(A_{i}, B_{i}, Q_{i}\right), i=1, \cdots, r_{d}$, is a finite family of parametrizing triples satisfying the condition (iv). Without loss of generality we can assume that $\left(A_{i}, B_{i}, Q_{i}\right), 1 \leqslant i \leqslant v_{d}$, for some $v_{d} \leqslant r_{d}$, form the set of all triples of our family $\left(A_{i}, B_{i}, Q_{i}\right), 1 \leqslant i \leqslant r_{d}$, for which the image of the associated parametrizing functor $B_{i} \longrightarrow \bmod R$ contains infinitely many nonisomorphic indecomposable modules. Then by Lemma 5 one can construct a set of parametrizing triples $W=\left(A_{i}^{\prime}\right.$, ind $A_{1}^{\prime}$,

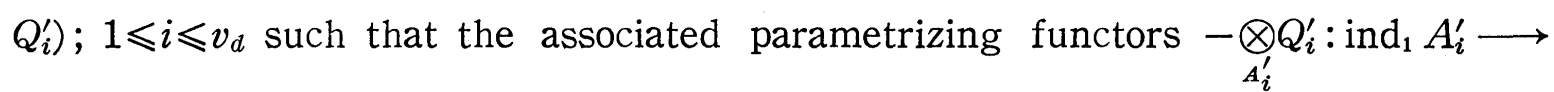
$\bmod R$ preserve indecomposables and there is only a finite number of nonisomor-

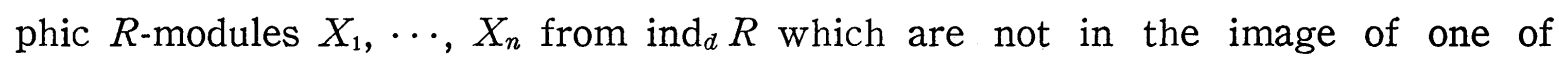
these functors. Thus the union $W \cup\left\{K\right.$, ind $\left.\left._{1} K, X_{j}\right) ; j=1, \cdots, n\right\}$ forms the required family of parametrizing triples.

The proof of $(\mathrm{v}) \longrightarrow(\mathrm{i})$ is an immediate consequence of Lemma 3 and Remark 1. Therefore the proposition is proved.

REMARK 2. Observe that Corollary 1 gives a possibility to express the definition of representation-tamness in terms of one parameter algebraic families of indecomposable modules (see [12]).

\section{The main result}

The main result of this paper is the following theorem.

THEOREM. Let $R$ be a locally bounded category over $K$. Then the following 
equivalences hold:

(i) $R$ is representation-tame if and only if any finite full subcategory $S$ of $R$ is representation-tame.

(ii) $R$ is representation-wild if and only if there exists a finite full subcategory $S$ of $R$ which is representation-wild.

In particular $R$ is either representation-wild or representation-tame but not simultaneously.

In order to prove the theorem we need two lemmas.

Lemma 6. Let $P$ be a full subcategory of a locally bounded category $R$. If $R$ is representation-tame then $P$ is so.

Proof. Consider the restriction functor $\operatorname{Res}: \operatorname{Mod} R \longrightarrow \operatorname{Mod} P$. It is wellknown $[1,13]$ that Res admits the full and faithful right adjoint functor $H: \operatorname{Mod} P$ $\longrightarrow \operatorname{Mod} R$ such that $\operatorname{Res} \circ H \cong i d_{\text {Mod } P}$, given by $H(N)(x)=\operatorname{Hom}_{P}(\operatorname{Res}(R(-, x)), N)$, for any $P$-module $N$ and object $x$ of $R$. Now let $d=\left(d_{y}\right)_{y \in P}$ be a finite dimensionvector in $P$. We will prove that there exists only a finite number of dimensionvectors $e=\left(e_{x}\right)_{x \in R}$ in $R$ such that $e=\operatorname{dim} H(N)$ for some indecomposable $P$-module $N$ with $\operatorname{dim} N=d$. It is enough to show the existence of a finite subcategory $S$ of $R$ and a natural number $m$ such that, for any indecomposable $P$-module $N$ with $\operatorname{dim} N=d$, supp $H(N)$ is contained in $S$ and $\operatorname{dim} H(N) \leqslant m$. Let $N$ be an indecomposable $P$-module with $\operatorname{dim} N=d$. Observe that if $H(N)(a)$ is nonzero then there is an object $x \in \operatorname{supp} N$ such that $R(x, a) \neq 0$, and therefore $a$ belongs to $\widehat{\operatorname{supp}} N$. But supp $N$ coincides with the full subcategory $D$ of $R$ formed by all objects $x$ such that $d_{x} \neq 0$. Then it is easy to see that $S=\hat{D}$ and

$$
m=\sum_{\substack{y \in D \\ x \in S}} d_{y} \cdot \operatorname{dim}_{K} R(y, x)
$$

have the required properties. Let $\left(K[T], \operatorname{ind}_{1} K[T], Q_{i}\right), i=1, \cdots, n$, be a finite family of parametrizing $\operatorname{ind}_{e} R$ for all $e$ as above. Then $\left(K[T], \operatorname{ind}_{1} K[T]\right.$, $\left.\operatorname{Res}\left(Q_{i}\right)\right)$, $i=1, \cdots, n$, is a finite family of parametrizing triples parametrizing ind ${ }_{d} P$ and the lemma is proved.

Lemma 7. Let $P$ be a full subcategory of a locally bounded category $R$. If $P$ is representation-wild then $R$ is so.

Proof. Let $F=-\underset{K\langle x, y\rangle}{\otimes} Q: \bmod K\langle x, y\rangle \longrightarrow \operatorname{Mod} P$ be a strict functor satisfying the conditions (1) and (2). Then $S=\operatorname{supp} Q$ is a finite full subcategory of $P$ of wild representation type. Denote by $I$ the ideal in $R$ generated by all objects of 
$R$ which do not belong to $\hat{S}$, that is, the ideal of all morphisms $f \in R(a, b), a, b \in R$, of the form $f=\sum_{j=1}^{n} h_{j} g_{j}$, for some $g_{j} \in R\left(a, c_{j}\right), h_{j} \in R\left(c_{j}, b\right)$ with $c_{j} \notin \hat{S}$. Then the quotient category $R / I$ is equivalent to a finite locally bounded category and the composition functor $L: S \longrightarrow R / I$ of the canonical embedding functor $S \longrightarrow R$ and the projection functor $R \longrightarrow R / I$ is full and faithful. Then by [6, Proposition 2], [5, Theorem 2], and Lemma 6, $R / I$ is representation-wild. Hence $R$ is representation-wild and the lemma is proved.

Proof of the Theorem. (i) Assume that any finite full subcategory of $R$ is representation-tame. We will show that $R$ is also representation-tame. Let $d=$ $\left(d_{x}\right)_{x \in R}$ be an arbitrary finite dimension-vector in $R$ and denote by $P$ the full subcategory of $R$ formed by all objects $x$ such that $d_{x} \neq 0$. Let $e=\left(e_{y}\right)_{y \in \hat{P}}$ be the dimension-vector in $\hat{P}$ defined by $e_{y}=d_{y}$ for any $y \in \hat{P}$. Then by [4, Lemma 2] the full subcategory of $\operatorname{Mod} R$ formed by all modules with support $P$ (resp. with dimension-vector $d$ ) is equivalent to the full subcategory of $\operatorname{Mod} \hat{P}$ formed by all modules with support $P$ (resp. with dimension-vector $e$ ). Then $\hat{P}$ is finite and if $\left(K[T]\right.$, ind $\left._{1} K[T], Q_{i}\right), i=1, \cdots, n_{e}$, is a finite family of parametrizing triples parametrizing $\operatorname{ind}_{e} \hat{P}$, then each $Q_{i}$ is a $K[T]$-R-bimodule and $\left(K[T]\right.$, $\left.\operatorname{ind}_{1} K[T], Q_{i}\right)$, $i=1, \cdots, n_{e}$, is a finite family of parametrizing triples parametrizing ind $d_{d} R$. The opposite implication in (i) follows from Lemma 6.

The statement (ii) follows directly from Lemma 7. Moreover, the last statement of the theorem is now an immediate consequence of $[5$, Theorem 2] and $[6$, Proposition 2].

COROllaRy 2. Let $R$ be a locally bounded category over $K$. Then $R$ is representation-tame if and only if, for any finite dimension-vector $d$ in $R$, ind $R$ is parametrized by a finite family of parametrizing triples $\left(A_{i}, B_{i}, Q_{i}\right), i=1, \cdots, n_{d}$, over $R$ such that $A_{i}=K$ or $A_{i}=K[T]_{h_{i}}, B_{i}=\operatorname{ind}_{1} A_{i}, Q_{i}$ are finitely generated free left $A_{i}$-modules, and the associated parametrizing functors $F_{i}: B_{i} \longrightarrow \bmod R$ are strict and preserve indecomposables.

Proof. In [6, Corollary 12] Drozd proved that a finite locally bounded $K$ category $R$ is representation-tame if and only if, for any finite dimension-vector $d$ in $R, \operatorname{ind}_{d} R$ is parametrized by a finite family of parametrizing triples $\left(A_{i}, B_{i}, Q_{i}\right)$, $i=1, \cdots, s_{d}$, over $R$ such that $A_{i}=K$ or $A_{i}=K[T]_{h_{i}}, B_{i}=\operatorname{ind}_{1} A_{i}, Q_{i}$ are finitely generated free left $A_{i}$-modules and the associated parametrizing functors $-\otimes_{i}: B_{i}$ $\longrightarrow \bmod R$ are strict. Then the corollary is a consequence of the Theorem and Lemma 5. 


\section{References}

[1] Auslander, M., Representation theory of artin algebras I, Comm. Algebra 1 (1974), $177-268$.

[2 ] Bongartz, K., Algebras and quadratic forms, J. London Math. Soc., (2) 28 (1983), 461469.

[ 3 ] Bongartz, K. and Gabriel, P., Covering spaces in representation theory, Invent. Math., 65 (1982), 331-378.

[4] Dowbor, P. and Skowroński, A., On Galois coverings of tame algebras, Archiv Math., (1985), 522-529.

[ 5 ] Drozd, Ju., On tame and wild matrix problems, In : Matrix problems, Kiev (1977), 104114 (In Russian).

[6] Drozd, Ju., Tame and wild matrix problems, In: Representations and quadratic forms, Kiev (1979), 29-74 (In Russian).

[7] Drozd, Ju., Tame and wild matrix problems, Representation theory II, Lecture Notes in Mathematics 832 (1980), 242-258.

[8] Gabriel, P., The universal cover of a representation-finite algebra, Representations of algebras, Lecture Notes in Mathematics 903 (1981), 68-105.

[9] Gabriel, P., Auslander-Reiten sequences and representation-finite algebras, Representation theory I, Lecture Notes in Mathematics 831 (1980), 1-71.

[10] Green, E. L., Group graded algebras and zero relation problem, Representations of algebras, Lecture Notes in Mathematics 903 (1981), 106-115.

[11] Hartshorn, R., Algebraic geometry, Springer-Verlag 1977.

[12] Kraft, H., Geometric methods in representation theory, Representations of algebras, Lecture Notes in Mathematics 944 (1982), 180-258.

[13] MacLane, S., Categories for the working mathematicians, Springer-Verlag 1971.

[14] Riedtmann, Ch., Algebren, Darstellungsköcher, Uberlagerungen und zurück, Comm. Math. Helv. 55 (1980), 199-224.

[15] Skowroński, A., The representation type of group algebras, In: Abelian groups and modules, Proceedings of the Udine Conference, Udine 1984, CISM No. 287, Springer-Verlag Wien-New York (1984), 517-531.

Institute of Mathematics,

Nicholas Copernicus University

Chopina 12/18, 87-100 Toruń, Poland 